\title{
Inequalities Pertaining Fractional Approach through Exponentially Convex Functions
}

\author{
Saima Rashid ${ }^{1,2, *}$, Muhammad Aslam Noor ${ }^{2}(\mathbb{D})$ and Khalida Inayat Noor ${ }^{2}(\mathbb{C}$ \\ 1 Department of Mathematics, Government College University, Faisalabad, Punjab 38000, Pakistan \\ 2 Department of Mathematics, COMSATS University Islamabad, Islamabad 44000, Pakistan \\ * Correspondence: saimarashid@gcuf.edu.pk
}

Received: 8 May 2019; Accepted: 26 June 2019; Published: 27 June 2019

check for updates

\begin{abstract}
In this article, certain Hermite-Hadamard-type inequalities are proven for an exponentiallyconvex function via Riemann-Liouville fractional integrals that generalize Hermite-Hadamard-type inequalities. These results have some relationships with the Hermite-Hadamard-type inequalities and related inequalities via Riemann-Liouville fractional integrals.
\end{abstract}

Keywords: convex function; exponentially-convex functions; Riemann-Liouville fractional integrals; Hermite-Hadamard inequality; power-mean inequality

MSC: 26D15; 26D10; 90C23

\section{Introduction}

Recently, many authors have participated in the development of the fractional calculus (differentiation and integration of arbitrary order). The applications of fractional calculus often appeared in fields such as generalized voltage dividers, engineering, capacitor theory, feedback amplifiers, electrode-electrolyte interface models, fractional order Chua-Hartley systems, fractional order models of neurons, the electric conductance of biological systems, fitting experimental data, medical, and analysis of special functions (see, e.g., [1-7]. The authors' interests concerned a variety of applications of fractional calculus in seemingly diverse fields of sciences and engineering (see, e.g., [8-10]). One may be referred to [11,12] for the details of the development of fractional calculus. The paper by Vladimir D. Zakharchenko and Ilya G. Kovalenko [13]: Best Approximation of the Fractional Semi-Derivative Operator by Exponential Series, considers the implementation of a fractional-differentiating filter of the order of $1 / 2$ by a set of automation astatic transfer elements, which greatly simplifies practical implementation. Real technical devices have the ultimate time delay, albeit small in comparison with the duration of the signal. As a result, the real filter will process the signal with some error.

Recently, Agulilar and co-authors [5-7] provided a new fractional operator. Fractional calculus is a term that refers to the integration and differentiation of arbitrary order. In other words, the meaning of the $k^{\text {th }}$ derivative $d^{k} y / d x^{k}$ and the $k^{\text {th }}$ iterated integral $\int \ldots \int d x$ is extended by considering a fractional $\alpha \in \mathbb{R}_{+}$parameter instead of the integer $k \in N$ parameter. Following this trend, some authors introduced new types of fractional derivatives and differences that allowed the appearance of the exponential function $[1,2]$ or Atangana-Baleanu fractional operator $[14,15]$ in the kernel of the operators, which makes it difficult to solve certain complicated fractional systems in their frames. Currently, a variety of fractional integral operators are under discussion, and many generalized fractional integral operators also take part in generalizing the theory of fractional calculus (see [1-15]).

The word "convexity" is the most important, natural, and fundamental notation in mathematics. Convex functions were presented by Johan Jensen over 100 years ago. Over the past few years, multiple 
generalizations and extensions have been made for convexity. These extensions and generalizations in the theory of inequalities have made valuable contributions in many areas of mathematics. Some new generalized concepts in this point of view are quasi-convex, strongly convex, approximately convex, logarithmically convex, mid-convex functions, pseudo-convex, $\phi$-convex, $\lambda$-convex, $h$-convex, delta-convex, Schur convex, and others [16-21].

A function $\varphi: I=[a, b] \subseteq \mathbb{R} \rightarrow \mathbb{R}$ is a convex function, if and only if it satisfies the inequality:

$$
\varphi\left(\frac{a+b}{2}\right) \leq \frac{1}{b-a} \int_{a}^{b} \varphi(x) \mathrm{d} x \leq \frac{\varphi(a)+\varphi(b)}{2}
$$

$a, b \in K$ with $a<b$, which is called the Hermite-Hadamard inequity. If $\varphi$ is concave on the interval $K$, then both inequalities in (1) hold in the reverse direction.

Recently, many researchers have given extensions, generalizations, refinements, variations, and applications $[16,17,21-29]$ for the Hermite-Hadamard inequality (1).

On the other hand, the minimum of the differentiable convex functions can be characterized by variational inequalities. These two aspects of the convexity theory have extensive applications and have provided effective tools for studying arduous problems. In recent years, integral inequalities have been derived via fractional analysis, which has emerged as another interesting technique. Due to advancement in inequalities, the comprehensive investigation of exponentially convex functions as the Riemann-Liouville fractional integral in the present paper is new. The class of exponentially-convex functions was introduced by Antczak [30] and Dragomir [31]. Inspired by these recent developments in convexity, Awan et al. [32] introduced and investigated another class of convex functions, which are called exponentially-convex functions and which is significantly different from the class introduced by $[30,31,33]$.

The proliferating research on big data analysis and deep learning has recently intensified the interest in information theory involving exponentially-convex functions. The smoothness of exponentially-convex functions is exploited for statistical learning, sequential prediction, and stochastic optimization; see $[30,34,35]$ and the references therein.

It is known [31] that a function $\varphi: I=[a, b] \subseteq \mathbb{R} \rightarrow \mathbb{R}$ is an exponentially convex function, if and only if it satisfies the inequality:

$$
e^{\varphi\left(\frac{a+b}{2}\right)} \leq \frac{1}{b-a} \int_{a}^{b} e^{\varphi(x)} d x \leq \frac{e^{\varphi(a)}+e^{\varphi(b)}}{2},
$$

$a, b \in K$ with $a<b$. The inequality (2), providing the upper and lower estimates for the exponential integral, is called the Hermite-Hadamard inequality.

The goal of this article is to establish Hermite-Hadamard-type inequalities for the Riemann-Liouville fractional integral using exponential convexity, as well as concavity for functions whose absolute values of the first derivative are convex. Here, we will derive a general integral inequality for the Riemann-Liouville fractional integral.

\section{Preliminaries}

Now, we recall and introduce some definitions for various convex functions.

Definition 1. A set $K \subset \mathbb{R}$ is said to be convex, if:

$$
t x+(1-t) y \in K, \quad \forall x, y \in K, t \in[0,1] .
$$

Definition 2. A function $\varphi: K \rightarrow \mathbb{R}$ is said to be a convex function, if and only if,

$$
\varphi(t x+(1-t) y) \leq t \varphi(x)+(1-t) \varphi(y), \quad \forall x, y \in K, t \in[0,1],
$$


and function $\varphi$ is called concave if $-\varphi$ is convex.

We now consider the class of exponentially-convex functions, which are mainly due to [30,31].

Definition 3 ([30,31]). A positive real-valued function $\varphi: K \subseteq \mathbb{R} \longrightarrow(0, \infty)$ is said to be exponentially convex on $\mathrm{K}$ if the inequality:

$$
e^{\varphi(t x+(1-t) y)} \leq t e^{\varphi(x)}+(1-t) e^{\varphi(y)}
$$

holds for $x, y \in K$ and $t \in[0,1]$.

Exponentially-convex functions are used to manipulate statistical learning, sequential prediction, and stochastic optimization; see $[30,34,35]$ and the references therein.

Using the novelties of Noor [22], one can study some aspects of exponentially-variational inequalities:

$$
\left\langle\left(e^{\varphi(x)}\right)^{\prime}, y-x\right\rangle=\left\langle\varphi^{\prime}(x) e^{\varphi(x)}, y-x\right\rangle \geq 0, \quad \forall y \in K .
$$

These types of inequalities are referred to as exponentially-variational inequalities and appear to be an interesting problem for further research. For the formulation, applications, and other aspects of variational inequalities, see [22-24].

Let us give some basic examples of exponentially-convex functions; for details, see [33].

(i) $\varphi(x)=c$ is exponentially convex on $(R)$ for any $c \geq 0$.

(ii) $\varphi(x)=e^{\alpha x}$ is exponentially convex on $(R)$ for any $\alpha \in \mathbb{R}$.

(iii) $\varphi(x)=x^{-\alpha}$ is exponentially convex on $(0, \infty)$ for any $\alpha>0$.

Let us recall an important definitions of the Riemann-Liouville fractional integral as follows:

Definition 4 ([4]). For $f \in L[a, b]$, the left-sided and right-sided Riemann-Liouville fractional integrals of order $\alpha>0$ with $a \geq 0$ are defined by:

$$
J_{a_{+}}^{\alpha} \varphi(x)=\frac{1}{\Gamma(\alpha)} \int_{a}^{x}(x-t)^{\alpha-1} \varphi(t) d t, \quad a<x,
$$

and:

$$
J_{b_{-}}^{\alpha} \varphi(x)=\frac{1}{\Gamma(\alpha)} \int_{x}^{b}(t-x)^{\alpha-1} \varphi(t) d t, \quad x<b,
$$

respectively, where $\Gamma(\alpha)$ is the Gamma function, and its definition is $\Gamma(\alpha)=\int_{0}^{\infty} e^{-u} u^{\alpha-1} d u$. It is to be noted that $J_{a^{+}}^{0} \varphi(x)=J_{b^{-}}^{0} \varphi(x)=\varphi(x)$; see [36].

In the case of $\alpha=1$, the fractional integral reduces to the classical integral. Properties concerning this operator can be found in [4].

\section{Main Results}

In the sequel of the paper, let $I \subset \mathbb{R}$ be a convex set in the finite-dimensional Euclidean space $\mathbb{R}^{n}$. From now on, we take $I=[\varsigma, \zeta]$, unless otherwise specified.

Before going on to our main result, first we prove the following integral inequality. 
Lemma 1. Let $I \subset \mathbb{R}$ be an open interval, $\varsigma, \zeta \in I$ with $\varsigma<\zeta$ and $\varphi:[\varsigma, \zeta] \rightarrow \mathbb{R}$ be a differentiable function such that $\left(e^{\varphi}\right)^{\prime}$ is integrable and $0<\alpha \leq 1$ on $(\varsigma, \zeta)$ with $\varsigma<\zeta$. If $\left|\left(e^{\varphi}\right)^{\prime}\right|$ is convex on $[\varsigma, \zeta]$, then the following identity holds:

$$
\begin{aligned}
& {\left[\left(\frac{(\zeta-\varsigma)^{\alpha}+(\zeta-x)^{\alpha}-(x-\varsigma)^{\alpha}}{(\zeta-\varsigma)^{\alpha}}\right) \frac{e^{\varphi}(\zeta)}{2}+\left(\frac{(\zeta-\varsigma)^{\alpha}+(x-\varsigma)^{\alpha}-(\zeta-x)^{\alpha}}{(\zeta-\varsigma)^{\alpha}}\right) \frac{e^{\varphi}(\varsigma)}{2}\right.} \\
& \left.-\frac{\Gamma(\alpha+1)}{2(\zeta-\varsigma)^{\alpha}}\left[J_{\zeta^{+}}^{\alpha} e^{\varphi(\zeta)}+J_{\zeta^{-}}^{\alpha} e^{\varphi(\varsigma)}\right]\right]=\frac{1}{2} \sum_{i=1}^{4} I_{i,}
\end{aligned}
$$

where:

$$
\begin{aligned}
& I_{1}=\frac{(x-\varsigma)^{\alpha+1}}{(\zeta-\varsigma)^{\alpha}} \int_{0}^{1}\left(\kappa^{\alpha}-1\right) e^{\varphi(\kappa x+(1-\kappa) \varsigma)} \varphi^{\prime}((\kappa x+(1-\kappa)) \varsigma) d \kappa, \\
& I_{2}=\frac{(\zeta-x)^{\alpha+1}}{(\zeta-\varsigma)^{\alpha}} \int_{0}^{1}\left\{\left(\frac{\varsigma-\zeta}{x-\zeta}-\kappa\right)^{\alpha}-\left(\frac{\varsigma-x}{x-\zeta}\right)^{\alpha}\right\} e^{\varphi(\kappa x+(1-\kappa) \zeta)} \varphi^{\prime}(\kappa x+(1-\kappa) \zeta) d \kappa, \\
& I_{3}=\frac{(\zeta-x)^{\alpha+1}}{(\zeta-\varsigma)^{\alpha}} \int_{0}^{1}\left(\kappa^{\alpha}-1\right) e^{\varphi(\kappa x+(1-\kappa) \zeta)} \varphi^{\prime}((\kappa x+(1-\kappa)) \zeta) d \kappa, \\
& I_{4}=\frac{(x-\varsigma)^{\alpha+1}}{(\zeta-\varsigma)^{\alpha}} \int_{0}^{1}\left\{\left(\left(\frac{\zeta-x}{x-\zeta}\right)^{\alpha}-\left(\frac{\zeta-\varsigma}{x-\varsigma}-\kappa\right)^{\alpha}\right\} e^{\varphi(\kappa x+(1-\kappa) \varsigma)} \varphi^{\prime}(\kappa x+(1-\kappa) \varsigma) d \kappa .\right.
\end{aligned}
$$

Proof. Using integration by parts and the change of variable technique, we have:

$$
\begin{aligned}
& I_{1}=\frac{(x-\varsigma)^{\alpha+1}}{(\zeta-\varsigma)^{\alpha}} \int_{0}^{1}\left(\kappa^{\alpha}-1\right) e^{\varphi(\kappa x+(1-\kappa) \varsigma)} \varphi^{\prime}((\kappa x+(1-\kappa)) \varsigma) d \kappa \\
& =\left.\frac{(x-\varsigma)^{\alpha+1}}{(\zeta-\varsigma)^{\alpha}}\left[\frac{\left(\kappa^{\alpha-1}\right) \varphi(\kappa x+(1-\kappa) \varsigma)}{(x-\varsigma)}\right]\right|_{0} ^{1}+\frac{\alpha}{x-\varsigma} \int_{0}^{1} \kappa^{\alpha-1} \varphi(\kappa x+(1-\kappa) \varsigma) d \kappa \\
& =\frac{(x-\varsigma)^{\alpha+1}}{(\zeta-\varsigma)^{\alpha}}\left[\frac{e^{\varphi(\varsigma)}}{x-\varsigma}-\frac{\alpha}{x-\varsigma} \int_{\zeta}^{x} \frac{(u-\varsigma)^{\alpha-1} e^{\varphi(\varsigma)}}{(x-\varsigma)^{\alpha}} d u\right] \\
& =\frac{(x-\varsigma)^{\alpha} e^{\varphi(\varsigma)}}{(\zeta-\varsigma)^{\alpha}}-\frac{\alpha}{x-\varsigma} \int_{\zeta}^{x}(u-\varsigma)^{\alpha-1} e^{\varphi(u)} d u \\
& I_{2}=\frac{(\zeta-x)^{\alpha+1}}{(\zeta-\varsigma)^{\alpha}} \int_{0}^{1}\left\{\left(\frac{\varsigma-\zeta}{x-\zeta}-\kappa\right)^{\alpha}-\left(\frac{\varsigma-x}{x-\zeta}\right)^{\alpha}\right\} e^{\varphi(\kappa x+(1-\kappa) \zeta)} \varphi^{\prime}(\kappa x+(1-\kappa) \zeta) d \kappa \\
& =\frac{(\zeta-x)^{\alpha+1}}{(\zeta-\zeta)^{\alpha}}\left[\left.\frac{\left(\left(\frac{\zeta-\zeta}{x-\zeta}-\kappa\right)^{\alpha}-\left(\frac{\zeta-x}{x-\zeta}\right)^{\alpha}\right)}{x-\zeta} e^{\varphi(\kappa x+(1-\kappa) \zeta)}\right|_{0} ^{1}\right. \\
& \left.+\frac{\alpha}{x-\zeta} \int_{0}^{1}\left(\frac{\varsigma-\zeta}{x-\zeta}-\kappa\right)^{\alpha-1} e^{\varphi(\kappa x+(1-\kappa) \zeta)} d \kappa\right] \\
& =\frac{(\zeta-x)^{\alpha+1}}{(\zeta-\zeta)^{\alpha}}\left[\frac{(\zeta-\zeta)^{\alpha}-(x-\zeta)^{\alpha} e^{\varphi(\zeta)}}{(\zeta-x)^{\alpha+1}}-\frac{\alpha}{(\zeta-x)^{\alpha+1}} \int_{x}^{\zeta}(u-\varsigma)^{\alpha-1} e^{f(u)} d u\right]
\end{aligned}
$$


Analogously:

$$
\begin{aligned}
I_{3} & =\frac{(x-\zeta)^{\alpha} e^{\varphi(\zeta)}}{(\zeta-\varsigma)^{\alpha}}-\frac{\alpha}{(\zeta-\varsigma)^{\alpha}} \int_{\zeta}^{x}(\zeta-u)^{\alpha-1} e^{f(u)} d u, \\
I_{4} & =\frac{(x-\varsigma)^{\alpha+1}}{(\zeta-\varsigma)^{\alpha}}\left[\frac{(\zeta-\varsigma)^{\alpha}-(x-\zeta)^{\alpha} e^{\varphi(\zeta)}}{(x-\varsigma)^{\alpha+1}}-\frac{\alpha}{(x-\varsigma)^{\alpha+1}} \int_{\zeta}^{x}(\zeta-u)^{\alpha-1} e^{f(u)} d u\right] .
\end{aligned}
$$

Adding the above identities, we get the required identity (3). This completes the proof.

In the following result, we investigate the fractional integral inequality that appears as the generalization and refinement of a well-known inequality for functions whose derivative in absolute value is exponentially convex.

Theorem 1. Let $\varphi: I=[\zeta, \zeta] \rightarrow \mathbb{R}$ be a differentiable function on the interior $I^{\circ}$ of $I$ with $\varsigma<\zeta$. If $\left(e^{\varphi}\right)^{\prime} \in(L[\varsigma, \zeta])$ and $0<\alpha \leq 1$ on $(\varsigma, \zeta)$ and if $\left|\left(e^{\varphi}\right)^{\prime}\right|$ is convex on $[\varsigma, \zeta]$, then the following fractional integral inequality holds:

$$
\begin{aligned}
& \mid\left(\frac{(\zeta-\varsigma)^{\alpha}+(\zeta-x)^{\alpha}-(x-\varsigma)^{\alpha}}{(\zeta-\varsigma)^{\alpha}}\right) \frac{e^{\varphi}(\zeta)}{2}+\left(\frac{(\zeta-\varsigma)^{\alpha}+(x-\varsigma)^{\alpha}-(\zeta-x)^{\alpha}}{(\zeta-\varsigma)^{\alpha}}\right) \frac{e^{\varphi}(\zeta)}{2} \\
& -\frac{\Gamma(\alpha+1)}{2(\zeta-\varsigma)^{\alpha}}\left[J_{\zeta^{+}}^{\alpha} e^{\varphi(\zeta)}+J_{\zeta^{-}}^{\alpha} e^{\varphi(\zeta)}\right] \mid \\
& \leq \frac{(x-\varsigma)^{\alpha+1}}{(\zeta-\varsigma)^{\alpha+1}}\left[\left(\theta_{1}+\delta_{1}\right)\left|e^{\varphi(x)} \varphi^{\prime}(x)\right|+\left(\theta_{2}+\delta_{2}\right)\left|e^{\varphi(\varsigma)} \varphi^{\prime}(\zeta)\right|+\left(\theta_{3}+\delta_{3}\right) \Delta(x, \zeta)\right] \\
& +\frac{(\zeta-x)^{\alpha+1}}{(\zeta-\varsigma)^{\alpha}}\left[\left(\theta_{1}+\rho_{1}\right)\left|e^{\varphi(x)} \varphi^{\prime}(x)\right|+\left(\theta_{2}+\rho_{2}\right)\left|e^{\varphi(\zeta)} \varphi^{\prime}(\zeta)\right|+\left(\theta_{3}+\rho_{3}\right) \Delta(x, \zeta)\right],
\end{aligned}
$$

where:

$$
\begin{aligned}
\theta_{1}= & \int_{0}^{1}\left|1-\kappa^{\alpha}\right| \kappa^{2} d \kappa=\frac{\alpha}{3(\alpha+3)^{\prime}} \\
\theta_{2}= & \int_{0}^{1}\left|1-\kappa^{\alpha}\right|(1-\kappa)^{2} d \kappa=\frac{\alpha\left(\alpha^{2}+6 \alpha+5\right)}{3(\alpha+1)(\alpha+2)(\alpha+3)^{\prime}}, \\
\theta_{3}= & \int_{0}^{1}\left|1-\kappa^{\alpha}\right| \kappa(1-\kappa) d \kappa=\frac{\alpha(\alpha+5)}{6(\alpha+2)(\alpha+3)}, \\
\delta_{1}= & \int_{0}^{1}\left|\left(\frac{\zeta-x}{x-\varsigma}\right)^{\alpha}-\left(\frac{\zeta-\zeta}{x-\zeta}-\kappa\right)^{\alpha}\right| \kappa^{2} d \kappa \\
= & \frac{2\left[(\zeta-x)^{\alpha+3}-(\varsigma-\zeta)^{\alpha+3}\right]}{(\alpha+1)(\alpha+2)(\alpha+3)(x-\varsigma)^{\alpha+3}}-\frac{2(\zeta-x)^{\alpha+2}}{(\alpha+1)(\alpha+2)(x-\varsigma)^{\alpha+2}} \\
& -\frac{(\zeta-x)^{\alpha+1}}{(\alpha+1)(x-\varsigma)^{\alpha+1}+\frac{(\zeta-x)^{\alpha}}{3(x-\varsigma)^{\alpha}},}
\end{aligned}
$$




$$
\begin{aligned}
& \delta_{2}=\int_{0}^{1}\left|\left(\frac{\zeta-x}{x-\varsigma}\right)^{\alpha}-\left(\frac{\zeta-\varsigma}{x-\varsigma}-\kappa\right)^{\alpha}\right|(1-\kappa)^{2} d \kappa \\
& =\frac{1}{3}\left(\frac{\zeta-x}{x-\varsigma}\right)^{\alpha}-\frac{(\zeta-\varsigma)^{\alpha+1}}{(x-\varsigma)^{\alpha+1}(\alpha+1)}-\frac{2(\zeta-\varsigma)^{\alpha+2}}{(\alpha+1)(\alpha+2)(x-\varsigma)^{\alpha+2}} \\
& +\frac{2\left[(\zeta-x)^{\alpha+3}-(\zeta-\zeta)^{\alpha+3}\right]}{(\alpha+1)(\alpha+2)(\alpha+3)(x-\zeta)^{\alpha+3}} \\
& \delta_{3}=\int_{0}^{1}\left|\left(\frac{\zeta-x}{x-\varsigma}\right)^{\alpha}-\left(\frac{\zeta-\varsigma}{x-\zeta}-\kappa\right)^{\alpha}\right| \kappa(1-\kappa) d \kappa \\
& =\frac{1}{6}\left(\frac{\zeta-x}{x-\varsigma}\right)^{\alpha}+\frac{(\zeta-x)^{\alpha+2}+(\zeta-\varsigma)^{\alpha+2}}{(\alpha+1)(\alpha+2)(x-\varsigma)^{\alpha+2}}+\frac{2\left[(\zeta-x)^{\alpha+3}-(\zeta-\varsigma)^{\alpha+3}\right]}{(\alpha+1)(\alpha+2)(\alpha+3)(x-\varsigma)^{\alpha+3}}, \\
& \rho_{1} \quad=\int_{0}^{1}\left|\left(\frac{\varsigma-\zeta}{x-\zeta}-\kappa\right)^{\alpha}-\left(\frac{\varsigma-x}{x-\zeta}\right)^{\alpha}\right| \kappa^{2} d \kappa \\
& =\frac{2\left[(\varsigma-\zeta)^{\alpha+3}-(\varsigma-x)^{\alpha+3}\right]}{(\alpha+1)(\alpha+2)(\alpha+3)(x-\zeta)^{\alpha+3}}-\frac{2(\varsigma-x)^{\alpha+2}}{(\alpha+1)(\alpha+2)(x-\zeta)^{\alpha+2}} \\
& -\frac{(\zeta-x)^{\alpha+1}}{(\alpha+1)(x-\zeta)^{\alpha+1}}-\frac{(\zeta-x)^{\alpha}}{3(x-\zeta)^{\alpha}}, \\
& \rho_{2} \quad=\int_{0}^{1}\left(\frac{\varsigma-\zeta}{x-\zeta}-\kappa\right)^{\alpha}-\left(\frac{\varsigma-x}{x-\zeta}\right)^{\alpha} \mid(1-\kappa)^{2} d \kappa \\
& =\frac{(\zeta-\zeta)^{\alpha+1}}{(x-\zeta)^{\alpha+1}(\alpha+1)}-\frac{2(\zeta-\zeta)^{\alpha+2}}{(x-\zeta)^{\alpha+2}(\alpha+1)(\alpha+2)} \\
& -\frac{2\left[(\zeta-x)^{\alpha+3}-(\zeta-\zeta)^{\alpha+3}\right]}{(x-\zeta)^{\alpha+3}(\alpha+1)(\alpha+2)(\alpha+3)}-\frac{1}{3}\left(\frac{\varsigma-x}{x-\zeta}\right)^{\alpha}, \\
& \rho_{3} \quad=\int_{0}^{1}\left(\frac{\varsigma-\zeta}{x-\zeta}-\kappa\right)^{\alpha}-\left(\frac{\varsigma-x}{x-\zeta}\right)^{\alpha} \mid \kappa(1-\kappa) d \kappa \\
& =\frac{(\varsigma-x)^{\alpha+2}+(\varsigma-\zeta)^{\alpha+2}}{(\alpha+1)(\alpha+2)(x-\zeta)^{\alpha+2}}-\frac{2\left[(\varsigma-x)^{\alpha+3}-(\varsigma-\zeta)^{\alpha+3}\right]}{(\alpha+1)(\alpha+2)(\alpha+3)(x-\zeta)^{\alpha+3}} \\
& -\frac{1}{6}\left(\frac{\varsigma-x}{x-\zeta}\right)^{\alpha} \text {, }
\end{aligned}
$$

and:

$$
\begin{aligned}
\Delta(x, \zeta) & =\left|e^{\varphi(\zeta)} \varphi^{\prime}(x)\right|+\left|e^{\varphi(x)} \varphi^{\prime}(\zeta)\right|, \\
\Delta(x, \zeta) & =\left|e^{\varphi(\zeta)} \varphi^{\prime}(x)\right|+\left|e^{\varphi(x)} \varphi^{\prime}(\zeta)\right| .
\end{aligned}
$$

Proof. Using Lemma 1, the property of the modulus, and the convexity of $\left|\left(e^{\varphi}\right)^{\prime}\right|$, we have:

$$
\begin{aligned}
& \mid\left[\left(\frac{(\zeta-\varsigma)^{\alpha}+(\zeta-x)^{\alpha}-(x-\varsigma)^{\alpha}}{(\zeta-\varsigma)^{\alpha}}\right) \frac{e^{\varphi}(\zeta)}{2}+\left(\frac{(\zeta-\varsigma)^{\alpha}+(x-\varsigma)^{\alpha}-(\zeta-x)^{\alpha}}{(\zeta-\varsigma)^{\alpha}}\right) \frac{e^{\varphi}(\zeta)}{2}\right. \\
& \left.-\frac{\Gamma(\alpha+1)}{2(\zeta-\varsigma)^{\alpha}}\left[J_{\zeta^{+}}^{\alpha} e^{\varphi(\zeta)}+J_{\zeta^{-}}^{\alpha} e^{\varphi(\zeta)}\right]\right]\left|=\frac{1}{2} \sum_{i=1}^{4}\right| N_{i} \mid,
\end{aligned}
$$


Now:

$$
\begin{gathered}
\left|N_{1}\right|=\frac{(x-\varsigma)^{\alpha+1}}{(\zeta-\varsigma)^{\alpha}} \int_{0}^{1}\left(\kappa^{\alpha}-1\right) e^{\varphi(\kappa x+(1-\kappa) \varsigma)} \varphi^{\prime}((\kappa x+(1-\kappa)) \varsigma) d \kappa \\
\leq \frac{(x-\varsigma)^{\alpha+1}}{(\zeta-\varsigma)^{\alpha}} \int_{0}^{1}\left|\left(1-\kappa^{\alpha}\right)\right|\left|e^{\varphi(\kappa x+(1-\kappa) \varsigma)} \varphi^{\prime}((\kappa x+(1-\kappa)) \varsigma)\right| d \kappa \\
\leq \frac{(x-\varsigma)^{\alpha+1}}{(\zeta-\varsigma)^{\alpha}} \int_{0}^{1}\left|\left(1-\kappa^{\alpha}\right)\right|\left\{\kappa\left|e^{\varphi(x)}\right|+(1-\kappa)\left|e^{\varphi(\varsigma)}\right|\right\}\left\{\kappa \mid \varphi^{\prime}(x)+(1-\kappa) \varphi^{\prime}(\varsigma)\right\} d \kappa \\
=\frac{(x-\varsigma)^{\alpha+1}}{(\zeta-\varsigma)^{\alpha}} \int_{0}^{1}\left|\left(1-\kappa^{\alpha}\right)\right|\left[\kappa^{2}\left|e^{\varphi(x)} \varphi^{\prime}(x)\right|+(1-\kappa)^{2}\left|e^{\varphi(\zeta)} \varphi^{\prime}(\varsigma)\right|\right. \\
\left.+\kappa(1-\kappa)\left\{\left|e^{\varphi(\varsigma)} \varphi^{\prime}(x)\right|+\left|e^{\varphi(x)} \varphi^{\prime}(\varsigma)\right|\right\}\right] d \kappa \\
=\frac{(x-\varsigma)^{\alpha+1}}{(\zeta-\varsigma)^{\alpha}} \int_{0}^{1}\left|\left(1-\kappa^{\alpha}\right)\right|\left[\kappa^{2}\left|e^{\varphi(x)} \varphi^{\prime}(x)\right|+(1-\kappa)^{2}\left|e^{\varphi(\varsigma)} \varphi^{\prime}(\varsigma)\right|\right. \\
+\kappa(1-\kappa) \Delta(x, \varsigma)] d \kappa \\
=\frac{(x-\varsigma)^{\alpha+1}}{(\zeta-\varsigma)^{\alpha}}\left[\theta_{1}\left|e^{\varphi(x)} \varphi^{\prime}(x)\right|+\theta_{2}\left|e^{\varphi(\varsigma)} \varphi^{\prime}(\varsigma)\right|+\theta_{3} \Delta(x, \varsigma)\right] ;
\end{gathered}
$$

similarly, we have:

$$
\begin{aligned}
\left|N_{2}\right| & =\frac{(\zeta-x)^{\alpha+1}}{(\zeta-\zeta)^{\alpha}} \int_{0}^{1}\left(\kappa^{\alpha}-1\right) e^{\varphi(\kappa x+(1-\kappa) \zeta)} \varphi^{\prime}((\kappa x+(1-\kappa)) \zeta) d \kappa \\
& \leq \frac{(\zeta-x)^{\alpha+1}}{(\zeta-\zeta)^{\alpha}} \int_{0}^{1}\left|\left(1-\kappa^{\alpha}\right)\right|\left|e^{\varphi(\kappa x+(1-\kappa) \zeta)} \varphi^{\prime}((\kappa x+(1-\kappa)) \zeta)\right| d \kappa \\
& =\frac{(\zeta-x)^{\alpha+1}}{(\zeta-\zeta)^{\alpha}} \int_{0}^{1}\left|\left(1-\kappa^{\alpha}\right)\right|\left\{\kappa^{2}\left|e^{\varphi(x)} \varphi^{\prime}(x)\right|+(1-\kappa)^{2}\left|e^{\varphi(\zeta)} \varphi^{\prime}(\zeta)\right|+\kappa(1-\kappa) \Delta(x, \zeta)\right] d \kappa \\
& =\frac{(\zeta-x)^{\alpha+1}}{(\zeta-\zeta)^{\alpha}}\left[\theta_{1}\left|e^{\varphi(x)} \varphi^{\prime}(x)\right|+\theta_{2}\left|e^{\varphi(\zeta)} \varphi^{\prime}(\zeta)\right|+\theta_{3} \Delta(x, \zeta)\right]
\end{aligned}
$$

where we have used the fact that, for $\alpha \in(0,1]$ and $\kappa \in[0,1]$,

$$
\begin{aligned}
\left|N_{3}\right| \leq & \frac{(x-\varsigma)^{\alpha+1}}{(\zeta-\varsigma)^{\alpha}} \int_{0}^{1}\left|\left(\frac{\zeta-x}{x-\varsigma}\right)^{\alpha}-\left(\frac{\zeta-\varsigma}{x-\varsigma}-\kappa\right)^{\alpha}\right|\left|e^{\varphi(\kappa x+(1-\kappa) \varsigma)} \varphi^{\prime}((\kappa x+(1-\kappa)) \varsigma)\right| d \kappa \\
\leq & \frac{(x-\varsigma)^{\alpha+1}}{(\zeta-\varsigma)^{\alpha}} \int_{0}^{1}\left|\left(\frac{\zeta-x}{x-\varsigma}\right)^{\alpha}-\left(\frac{\zeta-\varsigma}{x-\varsigma}-\kappa\right)^{\alpha}\right|\left[\kappa^{2}\left|e^{\varphi(x)} \varphi^{\prime}(x)\right|+(1-\kappa)^{2}\left|e^{\varphi(\varsigma)} \varphi^{\prime}(\varsigma)\right|\right. \\
& +\kappa(1-\kappa) \Delta(x, \varsigma)] d \kappa \\
\leq & \frac{(x-\varsigma)^{\alpha+1}}{(\zeta-\varsigma)^{\alpha}}\left[\delta_{1}\left|e^{\varphi(x)} \varphi^{\prime}(x)\right|+\delta_{2}\left|e^{\varphi(\varsigma)} \varphi^{\prime}(\zeta)\right|+\delta_{3} \Delta(x, \varsigma)\right] .
\end{aligned}
$$


Analogously:

$$
\begin{aligned}
\left|N_{4}\right| \leq & \frac{(\zeta-x)^{\alpha+1}}{(\zeta-\varsigma)^{\alpha}} \int_{0}^{1}\left|\left(\frac{\varsigma-\zeta}{x-\zeta}-\kappa\right)^{\alpha}-\left(\frac{\varsigma-x}{x-\zeta}\right)^{\alpha}\right|\left|e^{\varphi(\kappa x+(1-\kappa) \zeta)} \varphi^{\prime}((\kappa x+(1-\kappa)) \zeta)\right| d \kappa \\
\leq & \frac{(\zeta-x)^{\alpha+1}}{(\zeta-\zeta)^{\alpha}} \int_{0}^{1}\left|\left(\frac{\varsigma-\zeta}{x-\zeta}-\kappa\right)^{\alpha}-\left(\frac{\varsigma-x}{x-\zeta}\right)^{\alpha}\right|\left[\kappa^{2}\left|e^{\varphi(x)} \varphi^{\prime}(x)\right|+(1-\kappa)^{2}\left|e^{\varphi(\zeta)} \varphi^{\prime}(\zeta)\right|\right. \\
& +\kappa(1-\kappa) \Delta(x, \zeta)] d \kappa \\
\leq & \frac{(\zeta-x)^{\alpha+1}}{(\zeta-\zeta)^{\alpha}}\left[\rho_{1}\left|e^{\varphi(x)} \varphi^{\prime}(x)\right|+\rho_{2}\left|e^{\varphi(\zeta)} \varphi^{\prime}(\zeta)\right|+\rho_{3} \Delta(x, \zeta)\right],
\end{aligned}
$$

and suitable rearrangements complete the proof.

Theorem 2. Let $\varphi: I=[\zeta, \zeta] \rightarrow \mathbb{R}$ be a differentiable function on the interior $I^{\circ}$ of $I$ with $\zeta<\zeta$. If $\left(e^{\varphi}\right)^{\prime} \in(L[\zeta, \zeta])$ and $0<\alpha \leq 1$ on $(\zeta, \zeta)$ and if $\left|\left(e^{\varphi}\right)^{\prime}\right|^{q}$ is convex on $[\zeta, \zeta]$ for $q \geq 1$, then the following fractional integral inequality holds:

$$
\begin{aligned}
& \mid\left(\frac{(\zeta-\varsigma)^{\alpha}+(\zeta-x)^{\alpha}-(x-\varsigma)^{\alpha}}{(\zeta-\varsigma)^{\alpha}}\right) \frac{e^{\varphi}(\zeta)}{2}+\left(\frac{(\zeta-\varsigma)^{\alpha}+(x-\varsigma)^{\alpha}-(\zeta-x)^{\alpha}}{(\zeta-\varsigma)^{\alpha}}\right) \frac{e^{\varphi}(\zeta)}{2} \\
& \quad-\frac{\Gamma(\alpha+1)}{2(\zeta-\varsigma)^{\alpha}}\left[J_{\zeta^{+}}^{\alpha} e^{\varphi(\zeta)}+J_{\zeta^{-}}^{\alpha} e^{\varphi(\zeta)}\right] \mid \\
& \leq \frac{(x-\varsigma)^{\alpha+1}}{(\zeta-\varsigma)^{\alpha+1}}\left(\phi_{1}\right)^{1-\frac{1}{q}}\left[\theta_{1}\left|e^{\varphi(x)} \varphi^{\prime}(x)\right|+\theta_{2}\left|e^{\varphi(\varsigma)} \varphi^{\prime}(\zeta)\right|+\Delta_{1}(x, \varsigma)\right] \\
& \quad+\frac{(\zeta-x)^{\alpha+1}}{(\zeta-\varsigma)^{\alpha+1}}\left(\phi_{2}\right)^{1-\frac{1}{q}}\left[\theta_{1}\left|e^{\varphi(x)} \varphi^{\prime}(x)\right|+\theta_{2}\left|e^{\varphi(\zeta)} \varphi^{\prime}(\zeta)\right|+\Delta_{2}(x, \zeta)\right] \\
& \quad+\frac{(x-\varsigma)^{\alpha+1}}{(\zeta-\varsigma)^{\alpha+1}}\left(\phi_{3}\right)^{1-\frac{1}{q}}\left[\delta_{1}\left|e^{\varphi(x)} \varphi^{\prime}(x)\right|+\delta_{2}\left|e^{\varphi(\zeta)} \varphi^{\prime}(\zeta)\right|+\Delta_{1}(x, \varsigma)\right] \\
& \quad+\frac{(\zeta-x)^{\alpha+1}}{(\zeta-\varsigma)^{\alpha+1}}\left(\phi_{4}\right)^{1-\frac{1}{q}}\left[\rho_{1}\left|e^{\varphi(x)} \varphi^{\prime}(x)\right|+\rho_{2}\left|e^{\varphi(\zeta)} \varphi^{\prime}(\zeta)\right|+\Delta_{2}(x, \zeta)\right],
\end{aligned}
$$

where $\theta_{i}, \delta_{i}$, and $\rho_{i}$, for $i=1,2,3$, are given in Theorem 1 , and:

$$
\begin{aligned}
& \phi_{1} \quad=\int_{0}^{1}\left|\kappa^{\alpha}-1\right| d \kappa=\frac{\alpha}{\alpha+1}, \\
& \phi_{2} \quad=\int_{0}^{1}\left|1-\kappa^{\alpha}\right| d \kappa=\frac{\alpha}{\alpha+1} \\
& \phi_{3}=\int_{0}^{1}\left|\left(\frac{\zeta-x}{x-\varsigma}-\kappa\right)^{\alpha}-\left(\frac{\zeta-x}{x-\varsigma}\right)^{\alpha}\right| d \kappa \\
& =\frac{\left[(\zeta-x)^{\alpha+1}-(\zeta-\varsigma)^{\alpha+1}\right]-(\zeta-x)^{\alpha}(\alpha+1)(x-\varsigma)}{(\alpha+1)(x-\varsigma)^{\alpha+1}}, \\
& \phi_{4} \quad=\int_{0}^{1}\left|\left(\frac{\varsigma-\zeta}{x-\zeta}-\kappa\right)^{\alpha}-\left(\frac{\varsigma-x}{x-\zeta}\right)^{\alpha}\right| d \kappa \\
& =\frac{(x-\zeta)^{\alpha+1}-(\zeta-x)^{\alpha+1}-(\zeta-x)^{\alpha}(x-\zeta)(\alpha+1)}{(\alpha+1)(x+\zeta)^{\alpha+1}} \\
& \Delta_{1}(x, \varsigma)=\left[\left|e^{\varphi(x)} \varphi^{\prime}(\varsigma)\right|^{q}+\left.e^{\varphi(\varsigma)} \varphi^{\prime}(x)\right|^{q}\right],
\end{aligned}
$$




$$
\Delta_{2}(x, \zeta)=\left[\left|e^{\varphi(x)} \varphi^{\prime}(\zeta)\right|^{q}+\left.e^{\varphi(\zeta)} \varphi^{\prime}(x)\right|^{q}\right] .
$$

Proof. Using Lemma 1 and the property of the modulus, we have:

$$
\begin{aligned}
& \mid\left(\frac{(\zeta-\varsigma)^{\alpha}+(\zeta-x)^{\alpha}-(x-\varsigma)^{\alpha}}{(\zeta-\zeta)^{\alpha}}\right) \frac{e^{\varphi}(\zeta)}{2}+\left(\frac{(\zeta-\zeta)^{\alpha}+(x-\varsigma)^{\alpha}-(\zeta-x)^{\alpha}}{(\zeta-\varsigma)^{\alpha}}\right) \frac{e^{\varphi}(\varsigma)}{2} \\
& -\frac{\Gamma(\alpha+1)}{2(\zeta-\varsigma)^{\alpha}}\left[J_{\zeta^{+}}^{\alpha} e^{\varphi(\zeta)}+J_{\zeta^{-}}^{\alpha} \varphi^{\varphi(\zeta)}\right]\left|\leq \sum_{i=1}^{4}\right| R_{i} \mid,
\end{aligned}
$$

and using the convexity of $\left|\left(e^{\varphi}\right)^{\prime}\right|$, we have:

$$
\begin{aligned}
\left|R_{1}\right| \leq & \frac{(x-\varsigma)^{\alpha+1}}{(\zeta-\varsigma)^{\alpha+1}} \int_{0}^{1}\left(1-\kappa^{\alpha}\right)\left|e^{\varphi(\kappa x+(1-\kappa) \varsigma)} \varphi^{\prime}(\kappa x+(1-\kappa) \varsigma)\right| d \kappa \\
\leq & \frac{(x-\varsigma)^{\alpha+1}}{(\zeta-\varsigma)^{\alpha+1}}\left(\int_{0}^{1}\left(1-\kappa^{\alpha}\right) d \kappa\right)^{1-\frac{1}{q}}\left(\int_{0}^{1}\left(1-\kappa^{\alpha}\right)\left|e^{\varphi(\kappa x+(1-\kappa) \varsigma)} \varphi^{\prime}(\kappa x+(1-\kappa) \varsigma)\right|^{q}\right) d \kappa \\
= & \frac{(x-\varsigma)^{\alpha+1}}{(\zeta-\varsigma)^{\alpha+1}}\left(\phi_{1}\right)^{1-\frac{1}{q}}\left(\int_{0}^{1}\left(1-\kappa^{\alpha}\right) d \kappa\right)^{1-\frac{1}{q}}\left(\int_{0}^{1}\left(1-\kappa^{\alpha}\right)\left\{\kappa\left|e^{\varphi(x)}\right|^{q}+(1-\kappa)\left|e^{\varphi(\varsigma)}\right|^{q}\right\}\right. \\
\times & \left\{\kappa\left|\varphi^{\prime}(x)\right|^{q}+(1-\kappa)\left|\varphi^{\prime}(\varsigma)\right|^{q}\right\} d \kappa \\
= & \frac{(x-\varsigma)^{\alpha+1}}{(\zeta-\varsigma)^{\alpha+1}}\left(\phi_{1}\right)^{1-\frac{1}{q}}\left(\int_{0}^{1}\left(1-\kappa^{\alpha}\right) d \kappa\right)^{1-\frac{1}{q}}\left(\int _ { 0 } ^ { 1 } ( 1 - \kappa ^ { \alpha } ) \left\{\kappa^{2}\left|e^{\varphi(x)} \varphi^{\prime}(x)\right|^{q}+(1-\kappa)^{2}\left|e^{\varphi(\varsigma)} \varphi^{\prime}(\varsigma)\right|^{q}\right.\right. \\
& \left.+\kappa(1-\kappa)\left[\left|e^{\varphi(x)} \varphi^{\prime}(\varsigma)\right|^{q}+\left.e^{\varphi(\varsigma)} \varphi^{\prime}(x)\right|^{q}\right]\right\} d \kappa \\
= & \frac{(x-\varsigma)^{\alpha+1}}{(\zeta-\varsigma)^{\alpha+1}}\left(\phi_{1}\right)^{1-\frac{1}{q}}\left(\int_{0}^{1}\left(1-\kappa^{\alpha}\right) d \kappa\right)^{1-\frac{1}{q}}\left(\int _ { 0 } ^ { 1 } ( 1 - \kappa ^ { \alpha } ) \left\{\kappa^{2}\left|e^{\varphi(x)} \varphi^{\prime}(x)\right|^{q}\right.\right. \\
& \left.+(1-\kappa)^{2}\left|e^{\varphi(\varsigma)} \varphi^{\prime}(\varsigma)\right|^{q}+\kappa(1-\kappa) \Delta_{1}(x, \varsigma)\right\} d \kappa \\
= & \frac{(x-\varsigma)^{\alpha+1}}{(\zeta-\varsigma)^{\alpha+1}}\left(\phi_{1}\right)^{1-\frac{1}{q}}\left[\theta_{1}\left|e^{\varphi(x)} \varphi^{\prime}(x)\right|^{q}+\theta_{2}\left|e^{\varphi(\varsigma)} \varphi^{\prime}(\varsigma)\right|^{q}+\theta_{3} \Delta_{1}(x, \varsigma)\right] .
\end{aligned}
$$

Analogously:

$$
\begin{aligned}
& \left|R_{2}\right| \leq \frac{(\zeta-x)^{\alpha+1}}{(\zeta-\zeta)^{\alpha+1}}\left(\phi_{2}\right)^{1-\frac{1}{q}}\left[\theta_{1}\left|e^{\varphi(x)} \varphi^{\prime}(x)\right|^{q}+\theta_{2}\left|e^{\varphi(\zeta)} \varphi^{\prime}(\zeta)\right|^{q}+\theta_{3} \Delta_{2}(x, \zeta)\right], \\
& \left|R_{3}\right| \leq \frac{(x-\varsigma)^{\alpha+1}}{(\zeta-\varsigma)^{\alpha}} \int_{0}^{1}\left|\left(\frac{\zeta-x}{x-\varsigma}\right)^{\alpha}-\left(\frac{\zeta-\varsigma}{x-\varsigma}-\kappa\right)^{\alpha}\right|\left|e^{\varphi(\kappa x+(1-\kappa) \varsigma)} \varphi^{\prime}((\kappa x+(1-\kappa)) \zeta)\right| d \kappa \\
& \leq \frac{(x-\varsigma)^{\alpha+1}}{(\zeta-\varsigma)^{\alpha}}\left(\int_{0}^{1}\left|\left(\frac{\zeta-x}{x-\varsigma}\right)^{\alpha}-\left(\frac{\zeta-\varsigma}{x-\varsigma}-\kappa\right)^{\alpha}\right| d \kappa\right)^{1-\frac{1}{q}} \\
& \times\left(\int_{0}^{1}\left|\left(\frac{\zeta-x}{x-\zeta}\right)^{\alpha}-\left(\frac{\zeta-\varsigma}{x-\varsigma}-\kappa\right)^{\alpha}\right|\left|e^{\varphi(\kappa x+(1-\kappa) \varsigma)} \varphi^{\prime}((\kappa x+(1-\kappa)) \varsigma)\right|^{q} d \kappa\right. \\
& =\frac{(x-\varsigma)^{\alpha+1}}{(\zeta-\varsigma)^{\alpha}}\left(\phi_{3}\right)^{1-\frac{1}{q}}\left[\delta_{1}\left|e^{\varphi(x)} \varphi^{\prime}(x)\right|^{q}+\delta_{2}\left|e^{\varphi(\varsigma)} \varphi^{\prime}(\varsigma)\right|^{q}+\delta_{3} \Delta_{1}(x, \varsigma)\right]
\end{aligned}
$$


and:

$$
\begin{aligned}
\left|R_{4}\right| \leq & \frac{(\zeta-x)^{\alpha+1}}{(\zeta-\zeta)^{\alpha}} \int_{0}^{1}\left|\left(\frac{\zeta-\zeta}{x-\zeta}-\kappa\right)^{\alpha}-\left(\frac{\varsigma-x}{x-\zeta}\right)^{\alpha}\right| e^{\varphi(\kappa x+(1-\kappa) \zeta)} \varphi^{\prime}((\kappa x+(1-\kappa)) \zeta) \mid d \kappa \\
\leq & \frac{(\zeta-x)^{\alpha+1}}{(\zeta-\varsigma)^{\alpha}}\left(\int_{0}^{1}\left|\left(\frac{\zeta-\zeta}{x-\zeta}-\kappa\right)^{\alpha}-\left(\frac{\zeta-x}{x-\zeta}\right)^{\alpha}\right| d \kappa\right)^{1-\frac{1}{q}} \\
& \times\left(\int_{0}^{1}\left|\left(\frac{\varsigma-\zeta}{x-\zeta}-\kappa\right)^{\alpha}-\left(\frac{\varsigma-x}{x-\zeta}\right)^{\alpha}\right|\left|e^{\varphi(\kappa x+(1-\kappa) \zeta)} \varphi^{\prime}((\kappa x+(1-\kappa)) \zeta)\right|^{q} d \kappa\right. \\
= & \frac{(\zeta-x)^{\alpha+1}}{(\zeta-\varsigma)^{\alpha}}\left(\phi_{4}\right)^{1-\frac{1}{q}}\left[\rho_{1}\left|e^{\varphi(x)} \varphi^{\prime}(x)\right|^{q}+\rho_{2}\left|e^{\varphi(\zeta)} \varphi^{\prime}(\zeta)\right|^{q}+\rho_{3} \Delta_{2}(x, \varsigma)\right] .
\end{aligned}
$$

The proof is complete.

Remark 1. If we choose $q=1$, then under the assumptions of Theorem 2 , we get Theorem 1 in the present paper.

In the following, we obtain the bounds of the Hermite-Hadamard inequality for exponentiallyconcave functions.

Theorem 3. Let $\varphi: I=[\varsigma, \zeta] \rightarrow \mathbb{R}$ be a differentiable function on the interior $I^{\circ}$ of $I$ with $\zeta<\zeta$. If $\left(e^{\varphi}\right)^{\prime} \in(L[\varsigma, \zeta])$ and $0<\alpha \leq 1$ on $(\varsigma, \zeta)$. If $\left|\left(e^{\varphi}\right)^{\prime}\right|^{q}$ is concave on $[\varsigma, \zeta]$, for some fixed $p>1$ with $q=\frac{p}{p-1}$, then the following fractional integral inequality holds:

$$
\begin{aligned}
& \mid\left(\frac{(\zeta-\varsigma)^{\alpha}+(\zeta-x)^{\alpha}-(x-\varsigma)^{\alpha}}{(\zeta-\zeta)^{\alpha}}\right) \frac{e^{\varphi}(\zeta)}{2}+\left(\frac{(\zeta-\varsigma)^{\alpha}+(x-\varsigma)^{\alpha}-(\zeta-x)^{\alpha}}{(\zeta-\varsigma)^{\alpha}}\right) \frac{e^{\varphi}(\zeta)}{2} \\
& -\frac{\Gamma(\alpha+1)}{2(\zeta-\varsigma)^{\alpha}}\left[J_{\zeta^{+}}^{\alpha} e^{\varphi(\zeta)}+J_{\zeta^{-}}^{\alpha} e^{\varphi(\zeta)}\right] \mid \leq \frac{(x-\varsigma)^{\alpha+1}}{(\zeta-\varsigma)^{\alpha+1}}\left[\left|\phi_{1} e^{\varphi\left(\frac{Q_{1}}{\phi_{1}}\right)} \varphi^{\prime}\left(\frac{Q_{1}}{\phi_{1}}\right)\right|+\left|\phi_{3} e^{\varphi\left(\frac{Q_{3}}{\phi_{3}}\right)} \varphi^{\prime}\left(\frac{Q_{3}}{\phi_{3}}\right)\right|\right] \\
& \quad+\frac{(\zeta-x)^{\alpha+1}}{(\zeta-\varsigma)^{\alpha+1}}\left[\left|\phi_{2} e^{\varphi\left(\frac{Q_{2}}{\phi_{2}}\right)} \varphi^{\prime}\left(\frac{Q_{2}}{\phi_{2}}\right)\right|+\left|\phi_{4} e^{\varphi\left(\frac{Q_{4}}{\phi_{4}}\right)} \varphi^{\prime}\left(\frac{Q_{4}}{\phi_{4}}\right)\right|\right],
\end{aligned}
$$

where:

$$
\begin{aligned}
& Q_{1} \quad=\frac{\alpha^{2}(x+\varsigma)+\alpha(x+3 \zeta)}{2(\alpha+1)(\alpha+2)} \\
& Q_{2}=\frac{-\alpha^{2}(x+\zeta)-\alpha(x+3 \zeta)}{2(\alpha+1)(\alpha+2)} \\
& Q_{3} \quad=\frac{(x+\varsigma)(\zeta-x)^{\alpha}}{2(x-\varsigma)^{\alpha}}+\frac{x(\zeta-x)^{\alpha+1}-\varsigma(\zeta-\varsigma)^{\alpha+1}}{(x-\varsigma)^{\alpha+1}(\alpha+1)}-\frac{(\zeta-x)^{\alpha+2}-(\zeta-\varsigma)^{\alpha+2}}{(x-\varsigma)^{\alpha+1}(\alpha+1)(\alpha+2)^{2}} \\
& Q_{4} \quad=\frac{\zeta(\varsigma-\zeta)^{\alpha+1}-x(\varsigma-x)^{\alpha+1}}{(\alpha+1)(x-\zeta)^{\alpha+1}}-\frac{\left[(\varsigma-x)^{\alpha+2}-(\zeta-\zeta)^{\alpha+2}\right]}{(\alpha+2)(x-\zeta)^{\alpha+1}}-\frac{(\zeta-x)^{\alpha}(x+\zeta)}{2(x-\zeta)^{\alpha}}
\end{aligned}
$$

and:

$$
\Delta_{3}(y, z)=\left|e^{\varphi(y)} \varphi^{\prime}(z)\right|^{q}+\left|e^{\varphi(z)} \varphi^{\prime}(y)\right|^{q} .
$$

Proof. Using the concavity of $\left|\left(e^{\varphi}\right)^{\prime}\right|^{q}$ and the power-mean inequality, we obtain:

$$
\begin{aligned}
& \left|e^{\varphi(\kappa y+(1-\kappa) z)} \varphi^{\prime}(\kappa y+(1-\kappa) z)\right|^{q} \\
& \geq\left\{\kappa\left|e^{\varphi(y)}\right|^{q}+(1-\kappa)\left|e^{\varphi(z)}\right| q\right\}\left\{\kappa\left|\varphi^{\prime}(y)\right|^{q}+(1-\kappa)\left|\varphi^{\prime}(z)\right|^{q}\right\} \\
& \geq \kappa^{2}\left|e^{\varphi(y)} \varphi^{\prime}(y)\right|^{q}+(1-\kappa)^{2}\left|e^{\varphi(z)} \varphi^{\prime}(z)\right|^{q}+\kappa(1-\kappa)\left\{\left|e^{\varphi(y)} \varphi^{\prime}(z)\right|^{q}+\left|e^{\varphi(z)} \varphi^{\prime}(y)\right|^{q}\right\} \\
& =\kappa^{2}\left|e^{\varphi(y)} \varphi^{\prime}(y)\right|^{q}+(1-\kappa)^{2}\left|e^{\varphi(z)} \varphi^{\prime}(z)\right|^{q}+\kappa(1-\kappa) \Delta_{3}(y, z) .
\end{aligned}
$$


By the Jensen integral inequality and the concavity of $\left|\left(e^{\varphi}\right)^{\prime}\right|$, we have:

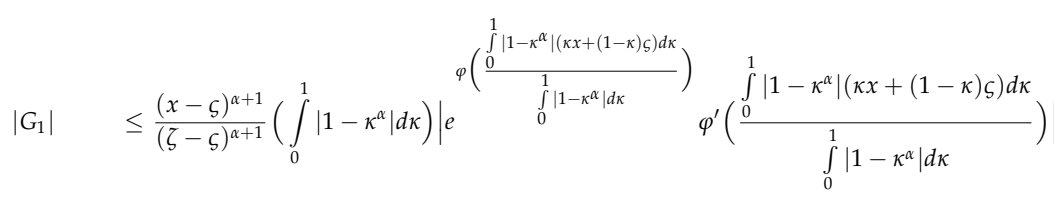

$$
\begin{aligned}
& =\frac{(x-\varsigma)^{\alpha+1}}{(\zeta-\varsigma)^{\alpha+1}}\left|\phi_{1} e^{\varphi\left(\frac{Q_{1}}{\phi_{1}}\right)} \varphi^{\prime}\left(\frac{Q_{1}}{\phi_{1}}\right)\right|,
\end{aligned}
$$

Analogously:

$$
\begin{aligned}
& \left|G_{2}\right| \leq \frac{(\zeta-x)^{\alpha+1}}{(\zeta-\zeta)^{\alpha+1}}\left|\phi_{2} e^{\varphi\left(\frac{Q_{2}}{\phi_{2}}\right)} \varphi^{\prime}\left(\frac{Q_{2}}{\phi_{2}}\right)\right|, \\
& \left|G_{3}\right| \leq \frac{(x-\varsigma)^{\alpha+1}}{(\zeta-\varsigma)^{\alpha+1}}\left(\int_{0}^{1}\left|\left(\frac{\zeta-x}{x-\varsigma}\right)^{\alpha}-\left(\frac{\zeta-\varsigma}{x-\varsigma}-\kappa\right)^{\alpha}\right| d \kappa\right) \\
& \times\left|e^{\varphi\left(\frac{\left.\int_{0}^{1}\left|\left(\frac{\zeta-x}{x-\zeta}\right)^{\alpha}-\left(\frac{\zeta-\zeta}{x-\zeta}-\kappa\right)^{\alpha}\right|(\kappa x+(1-\kappa))\right) d \kappa}{\int_{0}^{1}\left|\left(\frac{\zeta-x}{x-\zeta}\right)^{\alpha}-\left(\frac{\zeta-\zeta}{x-\zeta}-\kappa\right)^{\alpha}\right| d \kappa}\right)} \varphi^{\prime}\left(\frac{\int_{0}^{1}\left|\left(\frac{\zeta-x}{x-\zeta}\right)^{\alpha}-\left(\frac{\zeta-\zeta}{x-\zeta}-\kappa\right)^{\alpha}\right|(\kappa x+(1-\kappa) \zeta) d \kappa}{\int_{0}^{1}\left|\left(\frac{\zeta-x}{x-\zeta}\right)^{\alpha}-\left(\frac{\zeta-\zeta}{x-\zeta}-\kappa\right)^{\alpha}\right| d \kappa}\right)\right| \\
& =\frac{(x-\varsigma)^{\alpha+1}}{(\zeta-\varsigma)^{\alpha+1}}\left|\phi_{3} e^{\varphi\left(\frac{Q_{3}}{\phi_{3}}\right)} \varphi^{\prime}\left(\frac{Q_{3}}{\phi_{3}}\right)\right|, \\
& \left|G_{4}\right| \leq \frac{(\zeta-x)^{\alpha+1}}{(\zeta-\varsigma)^{\alpha+1}}\left(\int_{0}^{1}\left|\left(\frac{\varsigma-\zeta}{x-\zeta}-\kappa\right)^{\alpha}-\left(\frac{\varsigma-x}{x-\zeta}\right)^{\alpha}\right| d \kappa\right) \\
& \times\left|e^{\varphi\left(\frac{\int_{0}^{1}\left|\left(\frac{\zeta-\zeta}{x-\zeta}-\kappa\right)^{\alpha}-\left(\frac{\zeta-x}{x-\zeta}\right)^{\alpha}\right| \mid(\kappa x+(1-\kappa) \zeta) d \kappa}{\int_{0}^{1}\left|\left(\frac{\zeta-\zeta}{x-\zeta}-\kappa\right)^{\alpha}-\left(\frac{\zeta-x}{x-\zeta}\right)^{\alpha}\right| d \kappa}\right)} \varphi^{\prime}\left(\frac{\int_{0}^{1}\left|\left(\frac{\zeta-\zeta}{x-\zeta}-\kappa\right)^{\alpha}-\left(\frac{\zeta-x}{x-\zeta}\right)^{\alpha}\right| \mid(\kappa x+(1-\kappa) \zeta) d \kappa}{\int_{0}^{1}\left|\left(\frac{\zeta-\zeta}{x-\zeta}-\kappa\right)^{\alpha}-\left(\frac{\zeta-x}{x-\zeta}\right)^{\alpha}\right| d \kappa}\right)\right| \\
& =\frac{(\zeta-x)^{\alpha+1}}{(\zeta-\varsigma)^{\alpha+1}}\left|\phi_{4} e^{\varphi\left(\frac{Q_{4}}{\phi_{4}}\right)} \varphi^{\prime}\left(\frac{Q_{4}}{\phi_{4}}\right)\right|,
\end{aligned}
$$


where we have used the identities:

$$
\begin{aligned}
& Q_{1} \quad=\int_{0}^{1}\left|1-\kappa^{\alpha}\right||\kappa x+(1-\kappa) \varsigma| d \kappa=\frac{\alpha^{2}(x+\varsigma)+\alpha(x+3 \zeta)}{2(\alpha+1)(\alpha+2)}, \\
& Q_{2} \quad=\int_{0}^{1}\left|\kappa^{\alpha}-1\right||\kappa x+(1-\kappa) \zeta| d \kappa=\frac{-\alpha^{2}(x+\zeta)-\alpha(x+3 \zeta)}{2(\alpha+1)(\alpha+2)}, \\
& Q_{3} \quad=\int_{0}^{1}\left|\left(\frac{\zeta-x}{x-\varsigma}\right)^{\alpha}-\left(\frac{\zeta-\zeta}{x-\varsigma}-\kappa\right)^{\alpha}\right|(\kappa x+(1-\kappa) \varsigma) d \kappa \\
& =\frac{(x+\varsigma)(\zeta-x)^{\alpha}}{2(x-\varsigma)^{\alpha}}+\frac{x(\zeta-x)^{\alpha+1}-\zeta(\zeta-\zeta)^{\alpha+1}}{(x-\varsigma)^{\alpha+1}(\alpha+1)}-\frac{(\zeta-x)^{\alpha+2}-(\zeta-\zeta)^{\alpha+2}}{(x-\varsigma)^{\alpha+1}(\alpha+1)(\alpha+2)^{\prime}}, \\
& Q_{4} \quad=\int_{0}^{1}\left|\left(\frac{\varsigma-\zeta}{x-\zeta}-\kappa\right)^{\alpha}-\left(\frac{\varsigma-x}{x-\zeta}\right)^{\alpha}\right| \mid(\kappa x+(1-\kappa) \zeta) d \kappa \\
& =\frac{\zeta(\varsigma-\zeta)^{\alpha+1}-x(\varsigma-x)^{\alpha+1}}{(\alpha+1)(x-\zeta)^{\alpha+1}}-\frac{\left[(\zeta-x)^{\alpha+2}-(\zeta-\zeta)^{\alpha+2}\right]}{(\alpha+2)(x-\zeta)^{\alpha+1}}-\frac{(\varsigma-x)^{\alpha}(x+\zeta)}{2(x-\zeta)^{\alpha}} \text {. }
\end{aligned}
$$

\section{Conclusions}

In this article, we have derived a few inequalities of the Hermite-Hadamard-type for functions that possess first derivatives on the interior of an interval of real numbers, by utilizing the Hölder inequality and assumptions that the mappings $\left|\varphi^{\prime}\right|^{q}, q \geq 1$ are convex and concave. The resulting inequalities exhibited here surely give new bounds. For an appropriate and suitable choice of the value of $\alpha$ and $x=\frac{\varsigma+\zeta}{2}$, one can obtain several new and known results as special cases for various classes of convex functions and their variant forms. We can extend these results for different classes of exponentially-convex functions. These results have application in quantum physics, fractal geometry, and weather forecasting. It is expected that the ideas and technique of this paper may stimulate for further research in this fascinating field.

Author Contributions: Methodology, S.R.; Project administration, K.I.N.; Supervision, M.A.N. All authors worked jointly and contributed equally.

Acknowledgments: The authors are pleased to thank the Rector of COMSATSUniversity Islamabad, Islamabad, Pakistan, for providing excellent research and academic environments. The authors are grateful to the referees for their valuable and constructive suggestions.

Conflicts of Interest: The authors declare no conflict of interest.

\section{References}

1. Baleanu, D.; Guvenc, Z.B.; Machado, J.A.T. New Trends in Nanotechnology and Fractional Calculus Applications, 1st ed.; Springer: Berlin/Heidelberg, Germany, 2010.

2. Caponetto, R. Fractional Order Systems (Modelling and Control Applications); World Scientific: Singapore, 2010.

3. Hilfer, R. Applications of Fractional Calculus in Physics; World Scientific: Singapore, 2003.

4. Kilbas, A.; Srivastava, H.M.; Trujillo, J.J. Theory and Application of Fractional Differential Equations; North Holland Mathematics Studies; Elsevier: New York, NY, USA, 2006.

5. Gomez-Aguilar, J.F.; Torres, L.; Yepez-Martinez, H.; Baleanu, D.; Reyes, J.M.; Sosa, I.O. Fractional Lienard type model of a pipeline within the fractional derivative without singular kernel. Adv. Differ. Equ. 2016, 2016, 173. [CrossRef]

6. Morales-Delgado, V.F.; Taneco-Hernandez, M.A.; Gomez-Aguilar, J.F. On the solutions of fractional order of evolution equations. Eur. Phys. J. Plus 2017, 132, 47. [CrossRef] 
7. Yepez-Martineza, H.; Gomez-Aguilar, J.F.; Sosaa, I.O.; Reyesa, J.M.; Torres-Jimenez, J. The Feng's first integral method applied to the nonlinear mKdV space-time fractional partial differential equation. Rev. Mex Fisica 2016, 62, 310-316.

8. Caputo, M. Elasticita e Dissipazione; Zanichelli: Bologna, Italy, 1965.

9. Diethelm, K. The Analysis of Fractional Differential Equations, an Application Oriented, Exposition Using Differential Operators of Caputo Type; Lecture Notes in Mathematics; Springer: Heidelbereg, Germany, 2010.

10. Arqub, O.A. Solutions of time-fractional Tricomi and Keldysh equations of Dirichlet functions types in Hilbert space. Numer. Methods Partial. Differ. Equ. 2018, 34, 1759-1780. [CrossRef]

11. Arqub, O.A. Numerical solutions for the Robin time-fractional partial differential equations of heat and fluid flows based on the reproducing kernel algorithm. Int. J. Numer. Methods Heat. Fluid 2018, 28, 828-856. [CrossRef]

12. Arqub, O.A. Fitted reproducing kernel Hilbert space method for the solutions of some certain classes of time-fractional partial differential equations subject to initial and Neumann boundary conditions. Comput. Math. Appl. 2017, 73, 1243-1261. [CrossRef]

13. Zakharchenko, V.; Kovalenko, I. Best Approximation of the Fractional Semi-Derivative Operator by Exponential Series. Mathematics 2018, 6, 12. [CrossRef]

14. Arqub, O.A.; Al-Smadi, M. Atangana-Baleanu fractional approach to the solutions of Bagley-Torvik and Painleve equations in Hilbert space. Chaos Solitons Fractels 2018, 117, 161-167. [CrossRef]

15. Arqub, O.A.; Maayah, B. Numerical solutions of integrodifferential equations of Fredholm operator type in the sense of the Atangana-Baleanu fractional operator. Chaos Solitons Fractals 2018, 117, 117-124. [CrossRef]

16. Dragomir, S.S. Inequalities of Hermite-Hadamard type for $\phi$-convex functions on linear spaces. Preprint RGMIA Res. Rep. Coll. 2015, 87, 323-341.

17. Dragomir, S.S. Inequalities of Hermite-Hadamard type for $\lambda$-convex functions on linear spaces. Preprint RGMIA Res. Rep. Coll. 2014, 17, 18. [CrossRef]

18. Jensen, J.L.W.V. Om konvexe funktioner og uligheder mellem middlvaerdier. Nyt Tidsskrif for Matematik 1905, 16, 49-69.

19. Mangasarian, O.L. Pseudo-convex functions. J. Soc. Ind. Appl. Math. Ser. Cont. 1965, 3, 281-290. [CrossRef]

20. Rajba, T. On strong delta-convexity and Hermite-Hadamard type inequalities for delta-convex functions of higher order. Math. Inequal. Appl. 2015, 18, 267-293. [CrossRef]

21. Varosanec, S. On h-convexity. J. Math. Anal. Appl. 2007, 326, 303-311. [CrossRef]

22. Noor, M.A. General variational inequalities. Appl. Math. Lett. 1988, 1, 119-121. [CrossRef]

23. Noor, M.A. Some new approximation schemes for general variational inequalities. J. Math. Anal. Appl. 2000, 251, 217-229. [CrossRef]

24. Noor, M.A. Some developments in General Variational inequalities. Appl. Math. Comput. 2004, 152, $199-277$.

25. Rashid, S.; Noor, M.A.; Noor, K.I. Modified exponential convex functions and inequalities. J. Math. Theor. Phys. 2019, 2, 45-51.

26. Rashid, S.; Noor, M.A.; Noor, K.I. New estimates for exponentially convex functions via conformable fractional operators. Fractel Fract. 2019, 3, 19. [CrossRef]

27. Rashid, S.; Noor, M.A.; Noor, K.I. Some new generalizations for exponentially s-convex functions and inequalities via fractional operators. Fractel Fract. 2019, 3, 24. [CrossRef]

28. Sarikaya, M.Z.; Set, E.; Yaldiz, H.; Basak, N. Hermite-Hadamards inequalities for fractional integrals and related fractional inequalities. Math. Comput. Model. 2013, 57, 2403-2407. [CrossRef]

29. Qaisar, S.; Nasir, J.; Butt, S.I.; Asma, A.; Ahmad. F.; Iqbal, M.; Hussain, S. Some fractional integral inequalities of type Hermite-Hadamard through convexity. J. Inequal. Appl. 2019, 2019, 111. [CrossRef]

30. Antczak, T. On ( $p, r)$-invex sets and functions. J. Math. Anal. Appl. 2001, 263, 355-379. [CrossRef]

31. Dragomir, S.S.; Gomm, I. Some Hermite-Hadamard type inequalities for functions whose exponentials are convex. Stud. Univ. Babes-Bolyai Math. 2015, 60, 527-534.

32. Awan, M.U.; Noor, M.A.; Noor, K.I. Hermite-Hadamard inequalities for exponentially convex functions. J. Appl. Math. Inf. Sci. 2018, 12, 405-409. [CrossRef]

33. Pecaric, J.; Jaksetic, J. On exponential convexity, Euler-Radau expansions and stolarsky means. Rad Hrvatske Akademije Znanosti i Umjetnosti: Matematičke Znanosti 2013, 17, 81-94.

34. Alirezaei, G.; Mathar, R. On exponentially concave functions and their impact in information theory. J. Inf. Theor. Appl. 2018, 9, 265-274. 
35. Pal, S.; Wong, T.K.L. On exponentially concave functions and a new information geometry. Ann. Prob. 2018, 46, 1070-1113. [CrossRef]

36. Podlubni, I. Fractional Differential Equations; Academic Press: San Diego, CA, USA, 1999.

(C) 2019 by the authors. Licensee MDPI, Basel, Switzerland. This article is an open access article distributed under the terms and conditions of the Creative Commons Attribution (CC BY) license (http://creativecommons.org/licenses/by/4.0/). 